Aus dem pharmakologischen Institut zu Heidelberg.

\title{
Ueber die Undurchgängigkeit der Lunge für Ammoniak.
}

Von

R. Magnus.

Bei intravenöser Injection bewirken schon geringe Mengen von Ammoniak lebhafteste Erregung der Athmung, Krämpfe und Circulationsänderungen durch Reizung des Centralnervensystems (Funke u. Deahna ${ }^{1}$, Böhm u. Lange $\left.{ }^{2}\right)$. Um so mehr musste eine Beobachtung Knoll's ${ }^{3}$ ) auffallen, dass bei tracheotomirten und vagotomirten Thieren, bei denen also Reflexe durch sensible Reizung der Luftwege ausgeschlossen waren, auch durch länger dauernde Einathmung: concentrirter Ammoniakdämpfe keine Symptome, besonders keine Respirationsänderungen erzeugt wurden. Knoll, der hauptsächlich über die Reflexe von den Luftwegen auf die Athmung experimentirte, scheint aus diesen Versuchen damals geschlossen zu haben, dass die Ammoniakwirkung im Wesentlichen durch sensible Erregungen refiectorisch bedingt sei. Wir wissen heute, dass das nicht richtig ist. Es bleibt deshalb nur die Erklärung übrig, dass das Ammoniak in den Knoll'schen Versuchen gar nieht oder nur in geringer Menge aus der Athmungsluft ins Blut aufgenommen worden ist. Icb habe zur Aufklärung dieser Verhältnisse eine Reihe von Versuchen ausgeführt, und diese haben ergeben, dass in der That die Lunge für gasförmiges $\mathrm{NH}_{3}$ undurchgängig ist.

Es ist bekannt, wie ausserordentlich schnell die Aufnahme und Abgabe gasförmiger Substanzen in der Lunge erfolgt. Das lehrt ausser den Vorgängen bei dem normalen respiratorischen Gaswechsel besonders das blitzartige Einsetzen der Symptome nach Inhalation von Blausäure, und das Gleiche ergiebt sich für die Ausscheidung

1) Funke u. Deahna, Pfluger's arch.9.416. 1874.

2) Böhm u. Lange, Arch. f. exp. Path. u. Pharm. 2. 364. 1874.

3) Knoll, Sitzungsber. d. Wien. Akademie III. 68. 245. 1873. 
von Gasen in dem bekannten Vorlesungsversuch, bei welchem nach Injection von $\mathrm{H}_{2} \mathrm{~S}-\mathrm{Wasser}$ in die Jugularis die Schwärzung eines vor die Trachealeanüle gehaltenen Bleipapiers momentan auftritt. Um so deutlicher sind die Unterschiede, wenn man hiermit das Verhalten des Ammoniaks vergleicht.

Es wurde zunächst der Knoll'sche Versuch wiederholt:

Einem Kaninchen wurde eine tiefe Trachealfistel angelegt und beide Vagi am Halse durchschnitten. Das Thier athmete darauf durch M ïll er'sche Ventile. Das Inspirationsventil war mit starker Ammoniaklösung (70 g $\mathrm{NH}_{3}$ im Liter), das Exspirationsventil mit $\mathrm{H}_{2} \mathrm{SO}_{4}$ beschickt. Trotzdem nun das Thier $3 \mathrm{Minuten} \mathrm{lang} \mathrm{diese} \mathrm{starken} \mathrm{NH}_{3}$-Dämpfe einathmete, traten weder Krämpfe noch Aenderungen der Athmung ein. Nur vermehrte Secretion der Schleimbäute machte sich durch das Auftreten von Trachealrasseln bemerkbar. Darauf wurde das Inspirationsventil gewechselt nnd statt mit $\mathrm{NH}_{3}$ mit Schwefelwasserstoff gefüllt. Der Erfolg war, dass bereits 5 Secunden nach Beginn der $\mathrm{H}_{2} \mathrm{~S}$-Inhalation heftigste Krämpfe, und nach weiteren 15 Secunden der Exitus eintrat.

Hieraus ergiebt sich, dass bei der Inhalation von $\mathrm{NH}_{3}$-Dämpfen entweder gar nichts oder nur ganz geringe Mengen des giftigen Gases von den Lungen ins Blut aufgenommen werden.

Um aber zu erkennen, ob wirklich gar kein $\mathrm{NH}_{3}$ durch die Alveolarwände hindurchtreten kann, war es nöthig, den umgekehrten Weg einzuschlagen und das Auftreten, resp. Nichtanftreten von $\mathrm{NH}_{3}$ in der Exspirationsluft bei Anwesenheit freien Ammoniaks im Lungenblute zu untersuchen.

Dass die normale Exspirationsluft frei von $\mathrm{NH}_{3}$ ist, wurde durch vielfältige Untersuchungen festgestellt. Noch in jüngster Zeit konnte Formánek ${ }^{1)}$ zeigen, dass, wenn man Zersetzungsvorgänge an den Schleimhäuten durch sorgfältige Reinigung ausschliesst, die Ausathmungsluft keine Spur von $\mathrm{NH}_{3}$ enthält. Weiter geben $\mathrm{Seh}$ iffer ${ }^{2}$ ), sowie Böhm u. Lange ${ }^{3}$ ) an, dass nach intravenöser Einfuhr von Ammoniaksalzen kein $\mathrm{NH}_{3}$ exhalirt wird. Salaskin ${ }^{4}$ ) sah bei Hunden mit Eck'seher Fristel zu Zeiten, wo der Ammoniakgehalt des Blutes erhöht war, kein $\mathrm{NH}_{3}$ in der Athmungsluft erscheinen, und Biedl $\mathrm{u}$. W in terber $\mathrm{g}^{5}$ ) vermissten es ebenso in der Exspirationsluft eines Hundes, dem caustisches Ammoniak in kleiner Menge intravenös eingeführt war. Aus diesem Nichtauftreten des Gases schlossen Böhm und Lange, sowie Biedl und Winterberg, dass das $\mathrm{NH}_{3}$

1) Formánek, Arch. f. Hygiene. 38. 1. 1900.

2) Schiffer, Berl. klin. Wochenschr. 1872, Nr. 42.

3) a. a. 0 .

4) Salaskin, Z. f. physiolog. Chem. 25. 463.1898.

5) Biedl u. Winterberg, Pfüger's Arch. 88. 140. 1901. 
im kreisenden Blut fest gebunden sei, da es ja sonst nothwendig exhalirt werden müsse, und erstere konnten in der That zeigen, dass nach Einfuhr von kohlensaurem Ammoniak erst bei Erwärmung auf $80^{\circ}$ das $\mathrm{NH}_{3}$ aus dem Blute frei wurde.

Da demnach die normale Exspirationsluft ammoniakfrei ist, so musste sich ein etwaiger Durchtritt dieses Gases durch die Lunge mit Leichtigkeit nachweisen lassen. Man musste nur dafur sorgen, dass wirklich freies $\mathrm{NH}_{3}$ in dem Blute vorhanden ist, welches durch die Lungeneapillaren kreist, und dann die Ausathmungsluft untersuchen.

Letztere Bedingung glaube ich in den nachfolgend geschilderten Versuchen erfullt zu haben:

Kaninchen $1000 \mathrm{~g}$ athmet mittels einer Trachealcanüle durch Müller'sche Ventile, von denen das Exspirationsventil mit Nessler's Reagens beschickt wird. Unter Vermeidung von Ammoniakverdunstung: wird eine Spritze an die Jugularis angeschlossen, welche $5 \mathrm{ccm} \mathrm{0,35}$ Proc. kaustisches Ammoniak in Kochsalzlösung enthält.

$3 \mathrm{~h} 58 \mathrm{~m}$. Injection von $5 \mathrm{ccm}$. - Nessler's Reagens bleibt ungetrübt.

$4 \mathrm{~h}$. Zweite Injection von $5 \mathrm{ccm}$ derselben Lösung, zuerst langsam, dann sehr schnell. Erregung und beschleunigte Athmung. Reagens ungetrubt.

$4 \mathrm{~h} .5 \mathrm{~m}$. Injection von $1 \mathrm{ccm}$ starker $\mathrm{NH}_{3}$-Lösung (7 Proc.). Darauf noch 4 Respirationen, dann Exitus. Das Reagens im Ventil bleibt vollstänđig ungetrübt.

Bei der Section findet sich die Lunge scharlachroth verfärbt. Beim Anschneiden der grossen Gefässe zeigt das Blut starken $\mathrm{NH}_{3}$ Geruch. Darüber gehaltenes Lakmuspapier färbt sich blau.

Wenn auch dieser mehrfach mit gleichem Resultat wiederholte Versuch sehon beweist, dass bei der Anwesenheit von freiem $\mathrm{NH}_{3}$ im Blute trotzdem die Ausathmuugsluft davon frei bleibt, so suchte ich doch noch nach einer Anordnung, bei welcher der Zutritt freien Ammoniaks zu den Lungencapillaren noch sicherer und iuberzeugender garantirt wird. Zu diesem Zwecke wurde nach verschiedenen anderen Versuchen der Weg eingeschlagen, dass die $\mathrm{NH}_{3}-\mathrm{L}$ ösung direct in die Pulmonalarterie eingespritzt wurde.

Zu diesem Zwecke wurde dem chloralisirten Thier das Sternum in der Mitte gespalten und mit Haken auseinander gehalten, sodass das Herz offen dalag. Die künstliche Athmung erfolgte durch einen Blasebalg, und die Luft strich zuerst durch mehrere Waschflasehen mit $\mathrm{H}_{2} \mathrm{SO}_{4}$, um sie von $\mathrm{NH}_{3}$ zu befreien. Dann gelangte sie in die Lunge des Thieres. Die seitliche Oeffnung an der Trachealcanüle, aus der die Exspirationsluft entwich, war durch einen weiten Gummischlauch mit einem Ventil verbunden, welches mit Nessler's Reagens gefüllt war. 
Um die Empfindliehkeit der Anordnung zu prüfen, wurde zunäehst das Ventil mit einer Lösung von neutralem essigsauren Blei besehiekt und etwas $\mathrm{H}_{2} \mathrm{~S}$-Wasser einem Kaninehen intravenös injicirt. Die Schwärzung der Vorlage trat momentan ein. Ebenso entstand in einem mit Nessler's Reagens gefüllten Ventil sofort ein dichter Niedersehlag, als nur eine Spur $\mathrm{NH}_{3}$-haltiger Luft in die Schlauchleitung eingefïhrt wurde.

Zur Illustration der mehrfaeh angestellten Versuche mag folgendes Beispiel dienen:

Kaninchen $1810 \mathrm{~g}$, mit 0,6 pro $\mathrm{kg}$ Chloralhydrat narkotisirt. Künstliehe Athmung. Spaltung des Thorax.

Mit einer Pincette wird die Wurzel der Art. pulmonalis vorsichtig in die Höhe gehoben und die Hohlnadel einer $20 \mathrm{ccm}$ haltenden Spritze schräg in die Arterie eingestochen, um möglichst Blutung zu vermeiden.

5 h. $47 \mathrm{~m}$. Injection von $5 \mathrm{cem} \mathrm{NH}_{3}(0,35$ Proc.) in die Arteria pulmonalis. Heftigste Krämpfe und enorme Athembewegungen. Ne ss le r's Reagens absolut klar.

5 h. $49 \mathrm{~m}$. Injection von 5 eem $\mathrm{NH}_{3}$-Lösung. V orl ag e b l eib t klar.

$5 \mathrm{~h} .50 \mathrm{~m}$. Injection von $10 \mathrm{~cm}$. Vorlage bleibt klar.

Danach Exitus. 3 Minuten nach dem Tode trübt sich die Lösung bei andauernder künstlicher Athmung, zuerst langsam, dann immer sehneller bis zum dicken Niederschlag. Die Section ergiebt, dass die Lunge normales Aussehen besitzt und nach dem Herausnehmen stark nach $\mathrm{NH}_{3}$ riecht.

Dieser Versuch wurde mehrfach mit dem gleichen Erfolg wiederholt. Er demonstrirt in deutlicher Weise, dass auch bei directer Zufuhr freien Ammoniaks in die Lungengefässe kein $\mathrm{NH}_{3}$ in die Athmungsluft übergeht, er zeigt aber weiter, dass der Widerstand, den die Alveolarwände diesem Gas entgegensetzen, nur solange davert, als die Circulation durch die Lungengefässe stattfindet, und dass er einige Zeit nach dem Tode erliseht. Und da nun nach dem Tode ein Abdunsten von $\mathrm{NH}_{3}$ bei künstlicher Respiration nachweisbar war, ergiebt sich, dass anch während des Lebens das $\mathrm{NH}_{3}$ sicher in freiem verdunstungsfähigen Zustand im Lungenblute vorhanden war. Trotzdem trat während des Lebens keine Spur von Ammoniak dureh die Alveolarwand hindureh.

Die Angaben, welehe sich in der Literatur über das Verschwinden des Ammoniaks aus der Athmungsluft finden, widersprechen diesem Befunde nicht. So untersuchte K. B. Lehmann') die Exspirationsluft von Menschen, welche Luft mit $0,3 \% 0 \mathrm{NH}_{3}$ einathmeten. Er fand, dass 80-90 Proe. des $\mathrm{NH}_{3}$ nicht wieder aus-

1) K. B. Lehman n, Arch. f. Hygiene. 17. 324. 1893. 
geathmet wurden. Das beruht aber nach Lehmann's Untersuchungen anf der Absorption des Gases an den Schleimbäuten der obersten Luftwege, da bei nasaler Athmung Reizsymptome von Seiten des Kehlkopfs gering waren, solche von der Trachealschleimhant vollständig fehlten. Hier ist also das Gas gar nicht in die Lungen gelangt. Weiter konnte Gast ${ }^{1}$ ) in Lehmann's Laboratorium feststellen, dass aus einer $\mathrm{NH}_{3}$-haltigen Atmosphäre durch Hunde beträchtliche Mengen des Gases zum Verschwinden gebracht werden. Dasselbe geschah aber auch durch todte Hunde und durch Wolle, sodass dem Haarpelz der Thiere die Hauptrolle bei der Erscheinung zugeschrieben werden musste. Gast berechnet die Menge Ammoniak, welche überhaupt durch die Athmung höchstens absorbirt sein könnte, auf 10-20 Proc. der thatsächlich versehwundenen, nach meinen Versuchen ist sie, was wenigstens die Aufnahme durch die Lunge selbst betrifft, auf Null zu bewerthen. Aus Gast's Schilderung ergiebt sich ausserdem, dass seine Hunde trotz mehrstündigen Aufenthalts in der $\mathrm{NH}_{3}$ Atmosphäre nur Reizungssymptome von den Schleimhäuten, keine Zeichen von Ammoniakvergiftung aufwiesen.

Man muss daher nach dem vorliegenden experimentellen Material:

1. dem Fehlen des $\mathrm{NH}_{3}$ in der Expirationsluft von normalen und ammoniämischen Individuen,

2. dem Fehlen von Symptomen der allgemeinen Ammoniakvergiftung nach Einathmen von $\mathrm{NH}_{3}$-Dämpfen,

3. dem Nichterscheinen von $\mathrm{NH}_{3}$ in der Athmungsluft bei Anwesenheit freien Ammoniaks in den Lungencapillaren

die Undurchgängigkeit der Alveolarwand für Ammoniak als erwiesen betrachten.

Lassar ${ }^{2}$ ) hat bereits vor längerer Zeit angegehen, dass die Lunge für eingeathmete Säure- und Joddämpfe undurchgängig sei. Er konnte nach Einathmung von $\mathrm{HCl}_{3} \mathrm{H}_{2} \mathrm{SO}_{4}$, $\mathrm{HNO}_{3}$, $\mathrm{HJ}$ weder Allgemeinerscheinungen seiner Versuchsthiere, noch Aenderungen der Harnacidität feststellen. Ausserdem trat nach $\mathrm{HNO}_{3}$, HJ und $\mathrm{J}$ weder $\mathrm{HNO}_{3}$ noch $\mathrm{J}$ im Harn auf. Diese Versuche sind für die Salpetersäure von Spieg el ${ }^{3}$ ) nachgeprtift worden, wobei sich ergab, dass Einathmung von Salpetersäuredämpfen entgegen den Angaben von Lassar Allgemeinerscheinungen bewirkt, dass also eine Aufnahme der Dämpfe statthat, dass aber keine Nitrate im Harn auftreten, vermuthlich weil eine Umwandlung zu $\mathrm{NH}_{3}$ oder andren

1) Gast, Dissertation. Würzburg. 1899.

2) Lassar, Zeitschr. f. physiolog. Chemie. 1. 165.1877.

3) Spiegel, Dissertation. Würzburg 1894 (Kunkel). 
Verbindungen erfolgt. Für Jod liegen bis jetzt keine weiteren Untersuchungen vor.

Die oben geschilderten Experimente lehren, dass die Lunge durchaus nicht wahllos allen Gasen den Durchtritt gestattet. Ebenso wie wir am Darm und der Niere feststellen können, dass ihre Wände für bestimmte Körper entweder gar nicht oder schwer oder leicht permeabel sind, müssen wir a u ch der Alveolarwand die Eigenschaft zuschreiben, bestimmten Gasen den Durchtritt zu verwehren. Es ist daher nicht gestattet, die Aufnahme eines beliebigen Gases durch die Athmung als a priori sicher anzunehmen oder aus dem Nichtanftreten in der Exspirationsluft auf seine Abwesenheit im circulirenden Blute zu schliessen.

Anmerkung bei der Correctur: Ich habe inzwischen in der zoologischen Station in Neapel bei einigen Knochenfischen geprüft, ob ihre Kiemenmembran für $\mathrm{NH}_{3}$ durchgängig sei. $\mathrm{Zu}$ diesem $\mathrm{Z}$ wecke wurden die Fische, denen der Oesopbagus durch einen ejngeschobenen Korkstopfen abgeschlossen war, in eine dem Meerwasser isotonische Lösung von $\mathrm{NH}_{4} \mathrm{Cl}$ gesetzt. Darin gingen die Thiere in 10-15 Minuten zu Grunde. Dieses spricht dafür, dass $\mathrm{NH}_{3}-\mathrm{Salze}$ durch die Kiemen aufgenommen wurden. Da es jedoch sebr schwer zu entscheiden ist, ob der Stopfen den sehr dehnbaren Oesophagus wirklich wasserdicht verschlossen hat, möchte ich diese Versuche noch nicht als abschliessend ansehen. 